Klaus Rettinghaus (Berlin und Leipzig)

\title{
Zehn unbekannte Berlin-Briefe von Felix Mendelssohn Bartholdy
}

Mendelssohn war bekanntermaßen einer der produktivsten Briefschreiber des 19. Jahrhunderts. Obwohl im Laufe der Zeit etliche Briefe vernichtet wurden oder verloren gingen, ${ }^{1}$ hat sich eine schier unüberschaubare Anzahl - verstreut über die ganze Welt - bis heute erhalten. Und obschon nunmehr zahllose Publikationen einen Einblick in seine Korrespondenz gestatten, gilt es noch immer, unbekannte Briefe Felix Mendelssohn Bartholdys zu entdecken. Eine Auswahl solcher unveröffentlichter Briefe an Berliner Adressaten wird hier erstmals vorgestellt.

Die Briefe umfassen den Zeitraum von 1832 bis 1843 . Zum einen handelt es sich um Schreiben an Carl Friedrich Rungenhagen, den langjährigen Direktor der Sing-Akademie zu Berlin. Diese kleine Sammlung von ehemals neun Mendelssohn-Briefen befand sich neben anderen im Besitz von Dr. Wolfgang Schumann, ${ }^{2}$ der sich nach dem Zweiten Weltkrieg um die Rückgabe von Beständen der Sing-Akademie verdient gemacht hatte. Einer dieser Briefe aus dem Jahre 1841 wird seit 2002 im Bach-Archiv in Leipzig verwahrt, ${ }^{3}$ die übrigen acht befinden sich in Privatbesitz und sind bisher unveröffentlicht. Des Weiteren werden drei Schreiben präsentiert, die im Geheimen Staatsarchiv Preußischer Kulturbesitz verwahrt werden. Davon stehen zwei in Zusammenhang mit Mendelssohns Anstellung als Generalmusikdirektor für kirchliche und geistliche Musik und die damit verbundene Reorganisation des Berliner Domchors.

Alle Briefe sind hier chronologisch geordnet. Doppelte Bindestriche werden durch einfache wiedergegeben. Eindeutige Streichungen und Umformulierungen, die Mendelssohn teilweise während des Schreibens vornahm, werden nicht kommentiert.

Die acht Briefe an Rungenhagen enthalten größtenteils Anfragen bezüglich möglicher Teilnahmen an Proben der Sing-Akademie, so zum Beispiel in den ersten beiden hier wiedergegebenen Briefen.

\section{Mendelssohn an Carl Friedrich Rungenhagen in Berlin}

Berlin, Montag, den 10. September 1832

Hochgeehrter Herr Musikdirector

Sie waren vor 8 Tagen so gütig mir zu versprechen, daß ich zu Morgen die Erlaubniß erhalten sollte, zwei Fremde, die sehr wünschen die SingAcademie zu hören, mit dahin zu führen. Darf ich Sie nun um die Erfüllung Ihres Versprechens bitten? Sie werden beiden eine große Freude gewähren, da sie eifrige

1 Es ist zum Beispiel bekannt, dass Adolph Bernhard Marx die an ihn gerichteten Briefe vernichtete. Auch gingen einige auf Grund von Kriegseinwirkungen verloren.

2 Wolfgang Schumann (1904-1987), Sohn von Georg Schumann (1866-1952), der 1900 zum Direktor der Sing-Akademie zu Berlin gewählt wurde.

3 Brief vom 19. März 1841, D-LEb Rara II, 217; A 1075/1076. 
Musikfreunde sind u längst schon sich auf den Genuß freuen, u. Sie werden mir durch Ihre gütige Erlaubniß dazu einen großen Gefallen erzeigen.

Hochachtungsvoll

Ihr ergebenster

Berlin 10 Sept.

Felix Mendelssohn Bartholdy.

1832.

\section{Mendelssohn an Carl Friedrich Rungenhagen in Berlin}

Berlin, Dienstag, den 20. November 1832

Hochgeehrter Herr Musikdirector

Wären Sie wohl so gütig mir die Erlaubniß zu senden heut Abend zur Probe zwei Zuhörer mitzunehmen? Der eine davon hat sich für die Aufführungen schon abonnirt u wünscht das ihm fremde Werk 2 mal zu hören, der andre ist Musiker und nicht bemittelt genug um einen Platz zu kaufen. Ihrer gütigen Antwort entgegensehend

$$
\text { Ergebenst }
$$

$20 / 1132$

Felix Mendelssohn Bartholdy

Der nun folgende Brief befasst sich mit dem Orchestermaterial zu Händels Oratorium Israel in Egypt, das 1833 beim Niederrheinischen Musikfest in Düsseldorf unter Mendelssohns Leitung zur Aufführung kam. Auf die Anfrage des Festkomitees aus Düsseldorf ${ }^{4}$ vom 7. März 1833 antwortete Mendelssohn am 16. März aus Berlin: „[...] Wegen der Partitur und Orchesterstimmen von Israel in Egypten habe ich bei der Singakademie angefragt und es ist mir versprochen worden, mir dieselben für das Musikfest mitzugeben; nur wünscht man zu wissen wie lange die Stimmen dort gebraucht würden und wie bald sie wieder hier sein könnten. Wenn Sie also die Güte hätten, mir nur einige Zeilen an die Vorsteherschaft der Singakademie zuzusenden, in denen Sie sagen, binnen welcher Zeit die Musikalien zurück erfolgen sollen, so werde ich ihnen dieselben dann sogleich überschicken können oder selbst mitbringen. [...]"5 Anfang April, kurz vor seiner Abreise nach Düsseldorf, schrieb Mendelssohn diesbezüglich an Rungenhagen:

\section{Mendelssohn an Carl Friedrich Rungenhagen in Berlin}

Berlin, Montag, den 8. April 1833

Hochgeehrter Herr Musikdirector

Ich erhalte soeben den Auftrag Sie im Namen des Rheinischen Musikvereins zu ersuchen, die Orchesterstimmen welche Sie so gütig waren, dahin schicken zu wollen, sobald als möglich abzusenden, damit

4 GB-Ob M.D.M. d. 28, fol. 82, vgl. Susanna Großmann-Vendrey, Felix Mendelssohn Bartholdy und die Musik der Vergangenheit (= Studien zur Musikgeschichte des 19. Jahrhunderts 17), Regensburg 1969, S. 67.

5 Brief vom 16. März 1833, D-DÜhh, zitiert nach Großmann-Vendrey, S. 68. 
dieselben, wenn es sein kann, bis zum 18ten in Düsseldorf wären. Dürft ich Sie nun bitten, mich wissen zu lassen, ob Sie diesen Wunsch erfüllen können u. wann Sie das Packet dahin abgehn lassen wollen? Mit vollkommner Hochachtung

$$
\text { ergebenst }
$$

$8 / 433$

Felix Mendelssohn Bartholdy.

Im Notenarchiv des Städtischen Musikvereins Düsseldorf hat sich kein vollständiger Satz des Orchestermaterials zu Händels Oratorium erhalten. ${ }^{6}$

In den zwei folgenden Briefen kommt Mendelssohns Oratorium Paulus op. 36 zur Sprache. Der erste Brief ${ }^{7}$ bezieht sich auf eine Aufführung des Oratoriums durch die SingAkademie zu Berlin unter der Leitung von Rungenhagen am 18. Januar $1838 .{ }^{8}$ Im zweiten Brief, verfasst am 31. August 1841, fragt Mendelssohn nach spezifischem Orchestermaterial zu seinem Paulus.

\section{Mendelssohn an Carl Friedrich Rungenhagen in Berlin}

Leipzig, Donnerstag, den 18. Januar 1838

Hochgeehrter Herr Musikdirector

Ich danke Ihnen vielmal für die freundlichen Aeußerungen über meinen Paulus, welche Ihr geehrtes Schreiben vom 13ten Jan. enthält. Es ist mir die größte Freude zu erfahren, daß sich gerade dieses Werk der Theilnahme rühmen kann, die mir von allen die liebste ist, nämlich die der Musiker, deren Urtheil ich verehre, und ich kann daran sehen, daß mir manches darin besser gelungen ist, als ich selbst im Anfang dachte. Aber freilich bedarf mein Oratorium einer sehr sorgfältigen Ausführung, die Licht u. Schatten doppelt hervortreten macht, hin u. wieder die Mängel verdeckt, u. des Ganzen Eindruck erst harmonisch abrundet - Sie sagen mir in Ihrem Briefe, daß Sie auf eine solche bedacht gewesen sind, und so habe ich Ihnen auch dafür sehr vielen Dank zu sagen; bei den großartigen Mitteln, welche Ihnen in Berlin zu Gebot stehen kann das Gelingen der Aufführung dann gewiß nicht bezweifelt werden, u. es bleibt mir nur zu bedauern, daß es mir unmöglich war ihr selbst beizuwohnen u. daß ich den Genuß, der mir gewiß dadurch geworden wäre, verlieren mußte.

Mit vollkommner Hochachtung

$$
\text { ergebenst }
$$

Leipzig d. 18ten Januar

Felix Mendelssohn Bartholdy. 1838.

6 Vgl. Susanne Cramer, Die Musikalien des Düsseldorfer Musikvereins (1801-1929). Katalog, Stuttgart und Weimar 1996.

7 Mendelssohns Antwort auf einen Brief Rungenhagens vom 13. Januar 1838, GB-Ob M.D.M. d. 33, fol. 13.

8 Anders als oftmals zu lesen, war dies nicht die Berliner Erstaufführung des Oratoriums. Bereits am 13. September 1837 wurde Mendelssohns Paulus unter Julius Schneider in der Berliner Garnisonskirche zu Gunsten der Klein-Kinder-Bewahr-Anstalten aufgeführt, vgl. AmZ 39 (1837), Sp. 705 f. 


\section{Mendelssohn an Carl Friedrich Rungenhagen in Berlin}

Berlin, Dienstag, den 5. Januar 1841

Hochgeehrter Herr Musikdirector

Anbei übersende ich Ihnen, ein Schreiben an die Vorsteherschaft der Sing-Akademie, welches ich Sie an dieselbe gefälligst zu befördern bitte.

Zugleich erlaube ich mir Sie zu fragen, ob Sie vielleicht von meinem Paulus solche Baß (Orchester) Stimmen besitzen, in welchen Cello u. Contrabaß zusammengeschrieben sind? In den gedruckten sind sie separat. - Wäre das der Fall, so würde ich Ihnen sehr dankbar sein wenn Sie mir diese Baßstimmen durch Ueberbringer zuschicken $u$. auf einige Tage leihen wollten.

Hochachtungsvoll

$$
\text { ergebenst }
$$

Berlin d. 5ten Jan.

Felix Mendelssohn Bartholdy.

1841.

Die übrigen Briefe an Rungenhagen enthalten wieder Bitten und Dankesworte.

\section{Mendelssohn an Carl Friedrich Rungenhagen in Berlin}

Berlin, Dienstag, den 31. August 1841

Hochgeehrter Herr Musikdirector

Ich erlaube mir Sie zu der heut Abend Statt findenden Versammlung der Sing-Akademie um eine Einlaßcarte für meine Frau und mich ergebenst zu bitten.

Mit vollkommner Hochachtung

$$
\text { ergebenst }
$$

Berlin d. 31 Aug.

Felix Mendelssohn Bartholdy.

1841 .

\section{Mendelssohn an Carl Friedrich Rungenhagen in Berlin}

Berlin, Montag, den 6. September 1841

Hochgeehrter Herr Musikdirector

Abermals erlaube ich mir eine Bitte in Betreff der morgenden Versammlung der Sing-Akademie. Es ist ein Director der Englischen Musik Akademie, Mr. Hamilton hier, der für sich u seinen Reisegefährten eine Einlaßcarte wünscht, weil er um die musikalischen Merkwürdigkeiten Berlins zu kennen hergekommen ist. Wollten Sie den Wunsch dieses ausgezeichneten Mannes erfüllen, u. ihm durch den Ueberbringer 2 Carten zu kommen lassen so verbinden Sie dadurch aufs neue

Berlin d. 6ten Sept. 


\section{Mendelssohn an Carl Friedrich Rungenhagen in Berlin}

Berlin, Dienstag, den 30. November 1841

Hochgeehrtester Herr Musikdirector

Als ich gestern Abend nach Berlin zurückkehrte empfing ich Ihre freundlichen Zeilen und beeile mich Ihnen dafür meinen besten Dank zu sagen. Das erste Ihrer Concerte hat nun schon leider während meiner Abwesenheit Statt gefunden, doch hoffe ich gewiß, die nächstfolgenden nicht zu versäumen und Ihnen alsdann auch mündlich meinen Dank auszusprechen und die vollkommne Hochachtung mit welcher ich bin

Ew. Wohlgeboren

ergebenster Felix Mendelssohn Bartholdy.

Berlin d. 30st. Nov

1841.

Bei dem erwähnten Konzert handelt es sich vermutlich um das Händel'sche Oratorium Joseph, das die Sing-Akademie am 25. November aufgeführt hatte. ${ }^{9}$ Ein weiteres Konzert der Sing-Akademie in dieser Saison brachte dann am 10. Januar 1842 erneut Mendelssohns Paulus zur Aufführung, bei welcher der Komponist persönlich die Leitung übernahm. ${ }^{10}$

Mendelssohn war im November 1841 für einige Konzerte vorübergehend in Leipzig. Seinen Wohnsitz hatte er bereits im Juli des Jahres nach Berlin verlegt, um dem Ruf des preußischen Königs zu folgen. Seine Ernennung zum Kapellmeister und preußischen Generalmusikdirektor ist eng verbunden mit der Reorganisation des Königlichen Domchors in den Jahren 1842 und 1843. Damit in Verbindung stehende Vorgänge wurden beim preußischen Ministerium der Geistlichen-, Unterrichts- und Medizinal-Angelegenheiten in mehreren einzelnen Akten erfasst. So findet sich unter jenen auch Mendelssohns Berufungsakte. ${ }^{11}$ Diese Akte enthält u. a. zwei Briefe Mendelssohns an König Friedrich Wilhelm IV. Der erste Brief an den König vom 30. Juli 1841 wurde in diese Akte eingeordnet, da sie unterm 4. August 1841 an den Staatsminister Johann Albrecht Friedrich Eichhorn weitergeleitet worden war. Beim zweiten Brief vom 28. Oktober 1842 handelt es sich um eine autographe Abschrift, die Mendelssohn mit einem Begleitschreiben direkt an den Minister Eichhorn sandte.

Zwei nicht adressierte Briefe Mendelssohns vom 12. April $1842^{12}$ und vom 6. November 1842, die sich in den Akten des Ministeriums befinden, gingen wohl nicht, wie bislang angenommen, an den Geheimrat Ludwig von Massow. Mendelssohn schreibt als Anrede lediglich „Herr Geheimer Rath“, wohingegen er in Briefen an von Massow, wie auch in Schreiben an Graf Wilhelm von Redern und Minister Eichhorn stets die Anrede „Ew.

9 Vgl. AmZ 44 (1842), Sp. 65.

10 Ebd., Sp. 233 f.

11 D-Bga I. HA Rep. 76 Kultusministerium, Ve Sekt. 4 Abt. XV Nr. 164. Die Akte wurde erst 2004 wiedergefunden. Siehe dazu Thomas Ertelt und Jürgen Kloosterhuis, „Vorstellung eines wunderlichen jungen Mannes. Die Akte Mendelssohn“, in: Jahrbuch Preußischer Kulturbesitz 40 (2003), S. 277-302, sowie Wolfgang Dinglinger, „Acta betreffend: Die Berufung des Componisten Dr. Felix Mendelssohn Bartholdi nach Berlin. Briefe von und an Felix Mendelssohn Bartholdy", in: Mendelssohn-Studien 14 (2005), S. 189-219.

12 Dieses Schreiben wurde durch eine beglaubigte Abschrift vom März 1914 ersetzt. 
Excellenz" verwendet. ${ }^{13}$ Auch ist nicht ersichtlich, warum Briefe an von Massow in Akten des Kultusministeriums hätten eingeordnet werden sollen; eher ist anzunehmen, dass diese Briefe an den Geheimrat Karl Wilhelm Christian Kortüm ${ }^{14}$ gingen, der im Ministerium diese Vorgänge betreute.

Im geheimen Zivilkabinett des Königs wurden ebenfalls Akten angelegt, die sich auf den Königlichen Domchor beziehen; schließlich war der König die oberste Instanz des Chores. Der erste Band dieser Akten enthält Dokumente der Jahre 1838 bis 1853 und trägt den Titel „Acta / betr. / das Musik-Institut der Hof- und Domkirche / zu Berlin (Domchor.)“. ${ }^{15}$ Das Original des bereits oben erwähnten Schreibens von Mendelssohn an den König, das bisher nur in autographen Abschriften bekannt war, ${ }^{16}$ befindet sich in dieser Akte. Dieser Brief an den König, der hier nun folgt, birgt gegenüber den bekannten Abschriften keine Neuigkeiten, abgesehen von leichten Abweichungen bei gewissen Formulierungen. Bemerkenswert allerdings ist, dass der Brief einen Tag früher datiert als die Abschrift aus der Berufungs-Akte des Ministeriums.

\section{Mendelssohn an Friedrich Wilhelm IV. in Berlin ${ }^{17}$}

Berlin, Donnerstag, den 27. Oktober 1842

Allerdurchlauchtigster Großmächtigster König!

Allergnädigster König und Herr!

Ew. Königliche Majestät

haben in den unvergeßlichen Worten, welche Ew. Majestät an mich zu richten geruhten, eine so reiche Thätigkeit mir hier in Aussicht gestellt, und zugleich ein so ehrenvolles, so beglückendes Zutrauen mir ausgesprochen, daß ich den festen Entschluß gefaßt habe, meine Kräfte und Fähigkeiten, so lang ich lebe dem Dienste Ew. Königlichen Majestät zu weihen.

Ew. Majestät geruhten mir zu eröffnen, daß es die Absicht sei zu den schon bestehenden Königlichen Kirchenchören eine Anzahl tüchtiger Sänger hinzuzufügen, die für diese Chöre, sowie für die sich später etwa daran schließenden Gesangsliebhaber einen Kern bilden, und zum Anhaltspunct und Muster dienen solle, und durch welche die Kirchenmusik nach und nach zu erheben, zu veredeln und größerer Entwickelung entgegenzuführen sei. Um ferner den Gesang der Gemeinde auch durch Instrumente unterstützen zu können, woraus eine der schönsten, feierlichsten Wirkungen entsteht, wie Ew. Majestät sich namentlich von der Jubelfeier in der Nikolaikirche her erinnerten, solle eine kleine Anzahl Instrumentalisten (wahrscheinlich von den Mitgliedern des Königlichen Orchesters) ebenfalls als ein Kern für spätere, größere Aufführungen von Oratorien \&c., verpflichtet werden. Die Leitung eines auf solche Weise zu bildenden Musikerchores (einer ächten Königlichen Kapelle) erklärten Ew. Majestät mir dann anvertrauen, bis zu deren Organisation mir aber in Hinsicht meines Aufenthaltes vollkommne Freiheit gewähren zu wollen. Alle meine Wünsche für eine öffentliche musikalische Thätigkeit würden durch die Ausführung dieses Planes aufs herrlichste erfüllt, ich würde nie aufhören Ew. Majestät dankbar dafür zu sein, und zweifle nicht, daß die Organisation eines solchen Institutes ohne erhebliche Schwierigkeiten hier zu bewerkstelligen sein wird. Doch würde ich Ew. Majestät unterthänigst bitten diese Organisation nicht mir persönlich zu übertragen, sondern mir nur zu erlauben mit meiner Meinung und meinem Rath

13 Vgl. z. B. den Brief an Ludwig von Massow vom 21. Mai 1843, abgedruckt bei Richard Scheumann, „Briefe berühmter Komponisten aus dem Archiv des Königlichen Hof- und Domchors zu Berlin“, in: Mk. 8 (1908/1909), S. 259-270, hier: S. 260.

14 Karl Wilhelm Christian Kortüm (1787-1859), Geheimer Regierungsrat. Fast alle Schriftstücke in der Akte wurden von Kortüm gegengezeichnet.

15 D-Bga I. HA Rep. 89 Zivilkabinett, jüngere Periode, Nr. 23260.

16 Abschriften: D-Bga I. HA Rep. 76 Kultusministerium, Ve Sekt. 4 Abt. XV Nr. 164; S-Smf Sig. 2578.

17 D-Bga I. HA Rep. 89 Zivilkabinett, jüngere Periode, Nr. 23260, fol. 22-23. 
dabei mitzuwirken, wozu ich jederzeit aufs freudigste bereit sein werde. Bis nun also (um mich des eignen Ausdrucks Ew. Majestät zu bedienen) ein solches Instrument fertig wäre, auf welchem ich nachher zu spielen haben soll, würde ich von der Freiheit gebrauch machen dürfen, welche E. Majestät mir so gnädig bewilligten, und würde zunächst zur Direction der Abonnement-Concerte nach Leipzig zurückzukehren wünschen. Die Aufträge, welche Ew. Majestät mir zu Compositionen zu ertheilen geruhten werde ich dort mit der größten Liebe, nach meinen besten Kräften ausführen. Zugleich erbitte ich mir von Ew. Königlichen Majestät die Erlaubniß, da ich bis zur Organisation jenes Institutes zu keiner öffentlichen hiesigen Thätigkeit verpflichtet sein und völlige Freiheit genießen soll, auf die Hälfte des mir früher bewilligten Gehaltes Verzicht leisten zu dürfen, so lange ich diese Freiheit in Anspruch nehme.

Indem ich nochmals meinen innigsten Dank für alle Gnade ausspreche, welche Ew. Majestät mir in so vollem, reichlichen Maaße erwiesen haben ersterbe ich

Ew. Königlichen Majestät

unterthänigster

Berlin den 27ten October

Felix Mendelssohn Bartholdy.

1842.

Diesem Brief an den König liegt noch ein Begleitschreiben bei. In aller Regel las der König die an ihn gerichteten Briefe nicht selber. Die schriftlich vorgebrachten Anliegen wurden üblicherweise mündlich von einem Geheimrat des Zivilkabinetts vorgetragen. So schien es Mendelssohn wohl geboten, den sogenannten „vortragenden Rat“ - in diesem Fall wohl Kabinettsrat Karl Christian Müller ${ }^{18}$ - noch einmal gesondert auf einzelne Punkte hinzuweisen.

\section{Mendelssohn an Karl Christian Müller(?) in Berlin ${ }^{19}$}

Berlin, Freitag, den 28. Oktober 1842

Ew. Hochwohlgeboren

erlaube ich mir beiliegend den Brief an Sr. Majestät den König zu übersenden, welchen Sie, wie Sie mir sagten, morgen dem Könige vorzulegen die Güte haben wollten.

Zugleich erlaube ich mir noch einen Punct hervorzuheben, den einzigen welcher in unserm heutigen Gespräche übergangen und der auch, wie ich glaube, nicht deutlich genug in meinem Briefe an den König ausgesprochen ist; der nämlich, daß es die beiden Institute (nämlich des der Chorsänger und des der Instrumentalisten) zusammen sind, nicht eines derselben allein, wodurch meine Wünsche vollständig erfüllt sein würden[.]

Es möchten sich dem einen vorzugsweise Schwierigkeiten entgegenzustellen scheinen, und daher dann vielleicht der Gedanke entstehen, nur mit dem einen hervorzutreten, und das andre vor der Hand auf sich beruhen zu lassen. Dadurch würde aber nach meiner Meinung der schöne Plan des Königs nicht erfüllt, und dies ist es worauf ich besondern Werth legen würde.

Verzeihen Sie mir die nochmalige Belästigung mit den Details dieser Angelegenheit und genehmigen Sie vollkommenste Hochachtung mit welcher ich die Ehre habe zu sein

Ew. Hochwohlgeboren

ergebenster

Felix Mendelssohn Bartholdy.

Berlin d. 28 October

1842.

18 Karl Christian Müller (1773-1849), Geheimer Kabinettsrat, zu jener Zeit Chef der zweiten Abteilung des Zivilkabinetts.

19 Unveröffentlichter, nicht adressierter Brief, D-Bga I. HA Rep. 89 Zivilkabinett, jüngere Periode, Nr. 23260 fol. 21. 
Zuletzt noch ein Schreiben Mendelssohns, das in Abschrift ebenfalls im Geheimen Staatsarchiv verwahrt wird. Es findet sich als Beifügung eines Schreibens Conrad Löfflers vom 12. Dezember 1843 an den König. ${ }^{20}$ Darin bittet dieser um Erlaubnis zur Dedikation seiner „Ouverture über die russische Volkshymne“, welche im Manuskript ursprünglich beilag. ${ }^{21}$ Die Ouvertüre war den Ausführungen des Komponisten nach „im Saale des Königlichen Schauspielhauses zu Potsdam am 30ten November d. J. [1843]“ aufgeführt worden und erfreute sich eines „allgemeinen Beifalls“. Er verwies dabei auf „das in Abschrift ehrfurchtsvoll beigefügte Gutachten des Herrn General-Musik-Directors Ritters Dr. Felix Mendelssohn-Bartholdy“, dessen Original er „zur Zeit nicht in Händen habe.“ Der König nahm die Zueignung an. Der Entwurf des Antwortschreibens vom 2. Januar 1844 ist oben auf besagtem Brief vermerkt.

Löffler selbst hatte wenige Wochen zuvor eine Anfrage an Mendelssohn gerichtet, ${ }^{22}$ die zusammen mit einem weiteren Schreiben Löfflers ${ }^{23}$ in den sogenannten Green Books verwahrt wird. Er korrespondierte gelegentlich auch mit Richard Wagner und Giacomo Meyerbeer. Das besagte Gutachten von Mendelssohn lautet: ${ }^{24}$

\section{Abschrift.}

Daß mir mehrere der neusten Compositionen des Herrn Conrad Löffler, namentlich eine Ouverture über die russische Volkshymne, eine andre zu Nôtre dame de Paris, ein Streichquartett, einige Hefte Lieder und der Klavierauszug eines Singspiels bekannt sind, daß diese Werke überall von dem großen Fleiß und Eifer und von den schätzbaren Kenntnissen des Verfassers zeugen, daß besonders aus der Instrumentirung der Ouverturen genaue Bekanntschaft mit der Natur und der Wirkung der Blasinstrumente und Vertrautheit mit den Orchestereffecten hervorgeht, bescheinige ich hiermit nach bester Überzeugung. Felix Mendelssohn-Bartholdy.

Leipzig, den 31 Oktober.

1843 .

Löffler ging 1845 nach Wien, trat dort u. a. als „ein Schüler Mendelssohn's “25 auf und schrieb als Korrespondent für die Berliner musikalische Zeitung. Er wurde schließlich als musikalischer Scharlatan und Hochstapler enttarnt, der wohl nie ein eigenes Werk verfasst hatte, und ging nach Amerika. ${ }^{26}$ Bis das Original des Mendelssohn'schen Gutachtens auftaucht, muss daher dessen Authentizität in Zweifel gezogen werden.

20 D-Bga I. HA Rep. 89 Zivilkabinett, jüngere Periode, Nr. 20953 fol. 207-208. Die Lebensdaten von Conrad Löffler sind nicht zu ermitteln.

21 Der Standort des Manuskripts ist unbekannt. Es wird nicht in der Musiksammlung der Staatsbibliothek zu Berlin verwahrt und befand sich auch nicht im Schlossarchiv, vgl. Georg Thouret, Katalog der Musiksammlung auf der Königlichen Hausbibliothek im Schlosse zu Berlin, Leipzig 1895.

22 Unveröffentlichter Brief von Conrad Löffler vom 28. Oktober 1843, GB-Ob M.D.M. d. 49, fol. 251.

23 Unveröffentlichter Brief von Conrad Löffler vom 3. Mai 1846, GB-Ob M.D.M. d. 44, fol. 160.

24 Abschrift eines unveröffentlichten Zeugnisses für Conrad Löffler, D-Bga I. HA Rep. 89 Nr. 20953 fol. 206r.

25 Die Gegenwart 1 (1845), S. 184.

26 Vgl. Georg Richard Kruse, „August Conradi. Ein Gedenkblatt“, in: Mk. 48 (1913), S. 4-13. 